\title{
The genus Gnaptogaster Tobias (Hymenoptera: Braconidae: Gnamptodontinae) in Europe
}

\author{
Sergey A. Belokobylskij
}

Belokobylskij, S. A. 2007: The genus Gnaptogaster Tobias (Hymenoptera: Braconidae: Gnamptodontinae) in Europe. - Entomol. Fennica 18: 46-53.

The genera of the subfamily Gnamptodontinae and species of the genus Gnaptogaster Tobias are discussed. A new species, Gnaptogaster astrachanica sp. n., is described from the Astrakhan Province of Russia. Redescription of G. levipleuris Tobias, 1986, firstly recorded from Russia, is given. The genus Gnaptogaster is recorded for the first time for the faunas of Europe and Russia. Keys to genera of Gnamptodontinae and species of Gnaptogaster are provided.

S. A. Belokobylskij, Museum and Institute of Zoology Polish Academy of Science, Wilcza 64, 00-679 Warszawa, Poland; Zoological Institute Russian Academy of Sciences, St.Petersburg, 199034, Russia; E-mail: sb@zin.ru

Received 27 July 2005, accepted 25 April 2006

\section{Introduction}

The subfamily Gnamptodontinae is a small taxonomic group of the parasitic wasps that parasitise microlepidopteran larvae. This subfamily comprises 4 genera. The genus Gnamptodon Haliday, 1837 is the largest one, with more than 25 species. The species of this genus are distributed almost all over the World, but mainly in the Holarctic Region (van Achterberg 1983, 1988, Belokobylskij 1987, 1998, Tobias \& Saidov 1997, Chen et al. 2002).

The genus Pseudognaptodon Fischer, 1965 was initially described for 3 species from the Nearctic Region (Fischer 1965, 1967), and later (Belokobylskij 1993) was also recorded from the Oriental Region (Vietnam). The third genus, Neognamptodon Belokobylskij, 1999, with only the type species $N$. suturalis Belokobylskij, was recently described from Madagascar (Belokobylskij 1999).

Finally, the fourth gnamptodontine genus Gnaptogaster Tobias, 1976 (with type species $G$. mongolica Tobias, 1976) was originally found in Mongolia (Tobias 1976). Another species G. levipleuris Tobias, 1986 was later described also from the arid or semi-arid territories of East Kazakhstan (Tobias 1986). During a collecting trip in the semi-arid localities of south-east of the European part of Russia (Astrakhan Province), several specimens of the genus Gnaptogaster were collected. One specimen was determined as the male of Kazakhstanian G. levipleuris. Several other specimens belong to the new species described below, which is similar to Mongolian $G$. mongolica. Thus, the genus Gnaptogaster is recorded for the first time for the European as well as Russian faunas.

The hosts of the members of this subfamily are known only for the Gnamptodon species. Many Palaearctic species of this genus [such as G. pumilio (Nees), G. breviradialis Fischer, G. decoris (Foerster), G. georginae Achterberg etc.] are parasites of mining larvae of Nepticulidae with their pupation in the host cocoon (van Achterberg 1983, Belokobylskij 1987, 1998). It 
is quite possible that the hosts of Gnaptogaster are also mining microlepidopteran larvae.

The terms for wing venation are used as defined by Belokobylskij and Tobias (1998). The following abbreviations are used: POL postocellar line; OOL - ocular-ocellar line; Od maximum diameter of lateral ocellus; ZISP Zoological Institute, Russian Academy of Sciences (St. Petersburg, Russia).

\section{A key to genera of subfamily Gnamptodontinae}

1. Sternauli distinct and crenulate. Mesoscutum with long crenulate medioposterior furrow. Propodeum with median carina in basal half. Basal area of second tergite angulated medially. Brachial cell of fore wing completely closed. - Afrotropical Region

Neognamptodon Belokobylskij

- Sternauli absent or indistinct and smooth. Mesoscutum usually without medioposterior furrow (except some species of Gnaptogaster). Propodeum without median carina in basal half. Basal area of second tergite usually rounded medially. Brachial cell of fore wing at least partly (posteriorly or apico-posteriorly) open

2. Hypoclypeal cavity absent or very narrow. Radial cell of fore wing very strongly shortened. Dorsal carinae of first metasomal tergite entirely absent. Hind wing with recurrent vein. Maxillary palpi 5-segmented. Mesopleural sulcus usually shortly crenulate. - Palaearctic Region

Gnaptogaster Tobias

- Hypoclypeal cavity present and rather distinct. Radial cell of fore wing not shortened or distinctly (but not very strongly) shortened. Dorsal carinae of first metasomal tergite present at least basally. Hind wing usually without recurrent vein. Maxillary palpi 6-segmented. Mesopleural sulcus smooth

3

3. Second radiomedial vein of fore wing absent; only 1 radiomedial cell present. - Neotropical, Nearctic and Oriental Regions Pseudognaptodon Fischer

- Second radiomedial vein of fore wing present; 2 radiomedial cells present. - All World Gnamptodon Haliday

\section{Systematics}

Genus Gnaptogaster Tobias, 1976 (Figs 1, 2)

Type species: G. mongolica Tobias, 1976.

Tobias 1976: 319, 1986: 94; van Achterberg 1983: 26.

Description. Head distinctly transverse (Figs 1b, 2b). Antennal cavities widely separated. Hypoclypeal cavity absent or narrowly oval (Figs 1a, 2a). Clypeus weakly concave ventrally. Maxillary palpi 5-segmented; labial palpi 3-segmented. Notauli present in anterior 0.7 , very shallow or absent in posterior 0.3 (Fig. 1f). Mesoscutal pit absent or sometimes present (Fig. 1f). Prescutellar depression short and crenulate. Mesopleuron without or with smooth sternaulus (Fig. 1e). Pterostigma of fore wing wide, subtriangular (Figs 1j, 2h). Radial cell very strongly shortened. Radial vein with 2 (Fig. 1j) or 3 (Fig. 2h) abscissae. Second radiomedial vein present. Second radiomedial cell closed and small. Recurrent vein distinctly antefurcal. Discoidal cell petiolate. Nervulus postfurcal. Brachial cell widely open. In hind wing (Figs $1 \mathrm{k}, 2 \mathrm{i}$ ), first abscissa of mediocubital vein shorter than second abscissa; recurrent vein present, but strongly desclerotized. First metasomal tergite without dorsal carinae, with spiracles situated distinctly behind middle of tergite. Second tergite with large and distinctly delineated by deep furrow semi-oval basal area (Figs 1i, 2e). Second suture distinct and complete. Third tergite without basolateral areas. Ovipositor very short. Often vertex and at least part of metasoma with granulate sculpture.

\section{Gnaptogaster astrachanica sp. n. (Fig. 1)}

Type material. Holotype: 1 ( "Rossiya, Astrakhanskaya obl.[ast'], 60 km S Astrakhan', Dosang, zakrepl.[yonnye] peski, 22, 24.06.2004, S. Belokobylskij”.

Paratypes. $2 \bigcirc \bigcirc, 1 \jmath^{\lambda}$, with same label as holotype (ZISP); $1+$, same locality, "polupustynnaya step', 22.06.2004, Khalaim A.” (ZISP).

Description. Female. Body length 1.9-2.1 $\mathrm{mm}$; fore wing length $1.5-1.7 \mathrm{~mm}$.

Head distinctly transverse, its width about 


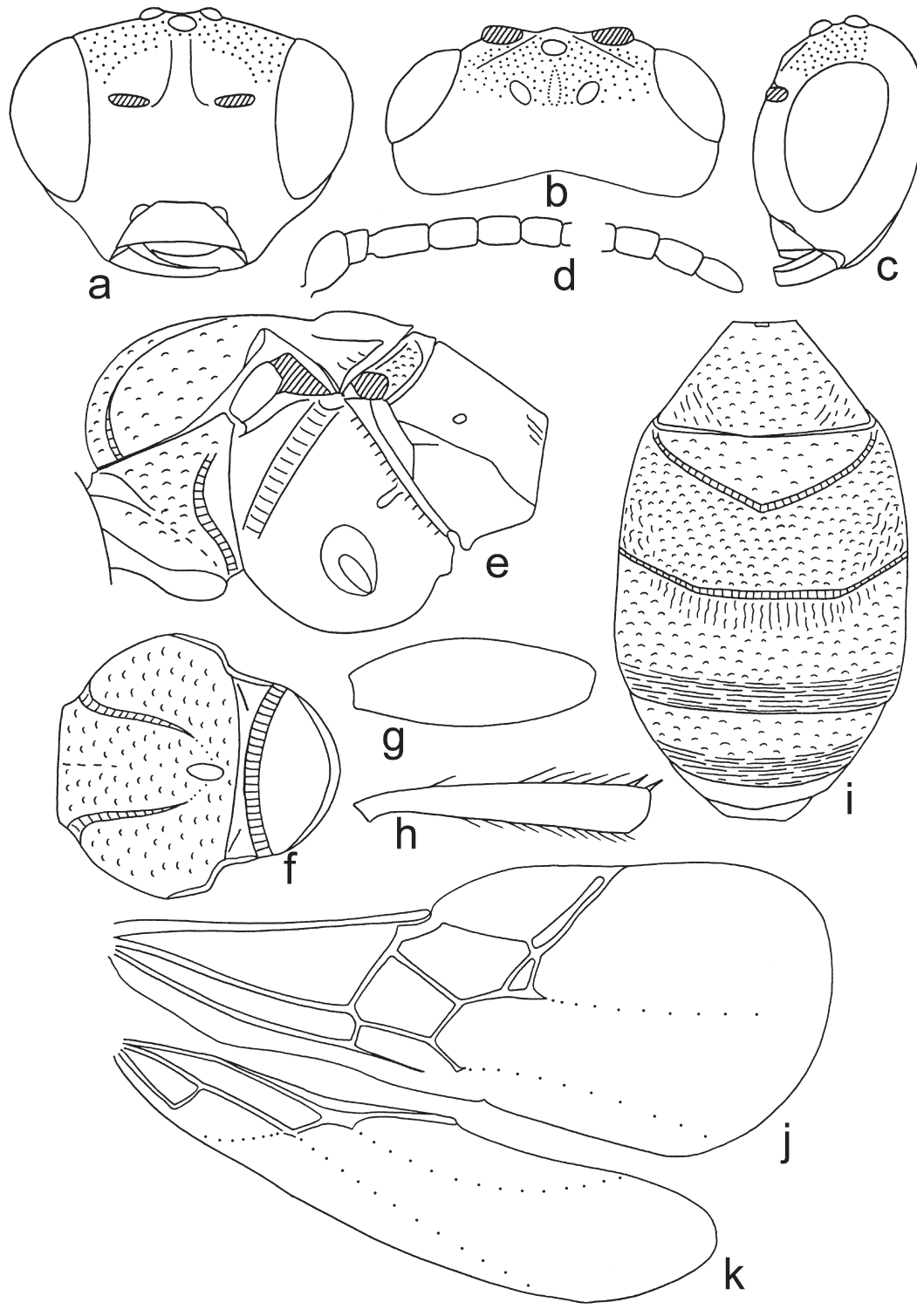

Fig. 1. Gnaptogaster astrachanica sp. n., holotype. - a. Head, front view. - b. Head, dorsal view. - c. Head, lateral view. - d. Basal and apical segments of antenna. - e. Mesosoma, lateral view. $-\mathrm{f}$. Mesonotum, dorsal view. - g. Hind femur. - h. Hind tibia. - i. Metasoma, dorsal view. $-\mathrm{j}$. Fore wing. $-k$. Hind wing. Scales: a-e, $g, h$ $-\mathrm{X} 126 ; \mathrm{f}, \mathrm{i}-\mathrm{X} 112 ; \mathrm{j}, \mathrm{k}$ $-X 98$. twice median length (dorsal view), 1.1-1.2 times width of mesoscutum. Head behind eyes (dorsal view) distinctly and roundly narrowed. Transverse diameter of eye (dorsal view) 2.3-2.5 times as long as temple (2.5-3.3 times for measure on straight line). Ocelli medium-sized, in triangle with base 1.3-1.4 times its sides. POL 1.7-2.1 times OD, almost equal to OOL. Furrow between hind ocelli present and shallow. Eye glabrous, 1.35-1.4 times as high as broad. Malar space height $0.25-0.3$ times height of eye, 0.8-0.9 times basal width of mandible. Hypostomal tooth (lateral view) absent. Face width 1.15-1.2 times height of eye and about twice height of face; face without middle ridge. Distance between antennal sockets 2.8-3.0 times diameter of socket, 3.7-4.0 times distance between socket and eye. Malar suture absent. Clypeus weakly convex, 2.7-3.0 times as wide as high, its ventral margin weakly concave. Mandible broad basally, uniformly narrowed towards apex, dorsal tooth distinctly longer than ventral tooth. Maxillary palpi about half as long as head height (without mandible).

Antennae rather thick, almost filiform, 16- 
18-segmented, 0.6 times as long as body. Scapus $1.4-1.5$ times as long as its maximum width. First flagellar segment 1.8-2.0 times as long as its apical width, 1.0-1.1 times as long as second segment. Median segments 1.6-1.7 times as long as wide. Penultimate segment 1.8 times as long as wide. Last segment pointed apically.

Mesosoma. Length 1.3-1.4 times its height, 1.5-1.6 times its width. Notauli distinct on anterior declivity and anterior 0.6 of horizontal part of mesoscutum, almost absent posteriorly, crenulate; medioposterior pit shallow, oval and rather short. Prescutellar depression deep, rather short, with numerous dense crenulae, $0.2-0.3$ times as long as scutellum. Scutellum convex, rather short and wide, 0.6 times as long as wide. Subalar depression deep, narrow, crenulate entirely or (sometimes narrowly) in upper half only. Sternauli rather deep, wide, subround, smooth, situated submedially on lower part of mesopleura. Propodeum without lateral tubercles, propodeal spiracles very small.

Wings. Fore wing 2.2-2.4 times as long as maximum width. Pterostigma subtriangular, wide, about twice as long as wide. Radial vein arising from middle of pterostigma. Radial cell very strongly shortened, very narrow, wider posteriorly than anteriorly; metacarpus very short, 0.04-0.07 times as long as pterostigma. Radial vein with 2 abscissae. First radial abscissa 0.15 0.2 times as long as maximum width of pterostigma. Second radial abscissa straight or very weakly curved, 7.5-8.0 times as long as first abscissa, 2.0-2.5 times as long as first radiomedial vein. Second radiomedial cell very short, subtriangular, 0.6-0.7 times as long as maximum width. Recurrent vein strongly antefurcal, 1.11.3 times as long as second abscissa of medial vein. Distance from nervulus to basal vein 0.5 0.6 times nervulus length. Brachial cell widely open posteroapically. Hind wing 3.6-3.7 times as long as wide. First costal abscissa $0.7-0.8$ times as long as second abscissa. First abscissa of mediocubital vein $0.7-0.8$ times as long as second abscissa. Recurrent vein unsclerotized, almost straight, oblique, directed towards base of wing, shortly antefurcal.

Legs. Hind femur wide, 2.6-2.8 times as long as wide. Hind tibia distinctly widened towards apex, with 3 long spines on outer apical margin.
Hind tarsus narrowed towards apex, 1.0-1.1 times as long as hind tibia. Basitarsus 0.6 times as long as second-fifth segments combined. Second tarsal segment $0.35-0.4$ times as long as basitarsus, 1.4-1.6 times as long as fourth segment, $0.7-0.8$ times as long as fifth segment (without pretarsus).

Metasoma 1.1-1.2 times as long as mesosoma. First tergite without or with small spiracular tubercles in posterior 0.3 , without lateral subapical furrows, strongly and evenly widened from base to apex. Maximum width of first tergite 2.7-3.5 times its minimum width; length 0.55 0.6 times its apical width. Second suture deep, narrow and crenulate. Basal area of second tergite distinctly delineated by deep and narrow furrow, medially as long as or a little shorter than half of tergite. Median length of second tergite 0.6-0.7 times its basal width, 1.1-1.2 times length of third tergite. Ovipositor sheath short, 0.6-0.7 times as long as first tergite, $0.17-0.18$ times as long as mesosoma, about 0.09 times as long as fore wing.

Sculpture and pubescence. Vertex smooth or very finely coriaceous, finely and densely granulate on sides of ocellar triangle; frons smooth or finely granulate-coriaceous; temple smooth; face smooth, but rather finely coriaceous below and sometimes laterally. Sides of pronotum very finely coriaceous, with narrow curved subvertical crenulate groove posteriorly. Mesoscutum almost smooth in anterior half, finely or very finely coriaceous in posterior half. Scutellum smooth or very finely coriaceous. Mesopleura smooth, sometimes very finely coriaceous below. Metapleura smooth or coriaceous in anterior 0.75 , rugose in posterior 0.25 . Propodeum finely or very finely coriaceous almost entirely. First metasomal tergite very finely coriaceous, sometimes with short striation anterolaterally. Second and third tergites rather distinctly or finely coriaceous, sometimes basal area almost smooth. Remainder tergites finely or very finely coriaceous with partly fine transverse striation. Vertex with rather dense short semi-erect setae. Frons bare, but with several setae laterally. Face with sparse semi-erect short setae. Mesoscutum mostly bare, with rather dense short setae anteriorly and posteriorly and sparse setae along notauli. Hind tibia with short, rather dense and semierect setae dorsally. 
Colour. Head black, clypeus and mandible brownish yellow, rarely face mostly reddish brown. Mesosoma light reddish brown, prothorax anteriorly and below, mesothorax ventrally, metathorax at most part or mainly dorsally, and propodeum usually apically dark reddish brown to black. Metasoma light reddish brown. Antennae black, scapus brownish yellow at most part. Palpi yellow. Tegulae yellow. Legs yellow or brownish yellow, all fifth tarsal segments black. Fore wing hyaline. Pterostigma yellow.

Male. Body length $1.8 \mathrm{~mm}$; fore wing length $1.4 \mathrm{~mm}$. Antennae longer, weakly setiform, 19segmented, 0.85 times as long as body. Median segments about twice as long as wide. Penultimate segment 2.4 times as long as wide. Notauli rather distinct posteriorly. Mesoscutum medioposteriorly with fine striation around notauli. First metasomal tergite finely rugose-striate with coriaceous sculpture, only coriaceous basomedially. Second and third tergites finely rugulose with fine granulation. Mesosoma black, with reddish: anterolateral spots, rather large medioposterior spot on mesoscutum, and scutellum mostly. Metasoma reddish brown, dark reddish brown partly, widely brownish yellow laterally. Hind tibia infuscate in posterior one-third. Otherwise similar to female.

Diagnosis. This new species is similar to $G$. mongolica Tobias (Mongolia) and differs in having the radial vein with 2 abscissae, the last radial abscissa straight and slender, the shape of second radiomedial cell subtriangular, the pterostigma yellow, the basal area of second tergite about as long as half of tergite, the metasoma entirely finely but distinctly sculptured, the antenna short, the mesoscutum medioposteriorly with short and sparse setae, and the mesosoma of female mostly light reddish brown.

Distribution. Russia (south-east of the European part).

\section{Gnaptogaster levipleuris Tobias, 1986 (Fig. 2) Gnaptogaster levipleuris Tobias 1986: 94.}

Material examined. Kazakhstan: $29 q$ (holotype and paratype), "peski Taskum, vost.[ochnee] Zaysana, Tobias, 25 V [1]961”. Russia: 10̄, Astrakhan Province, 60 km N Astrakhan', Dosang, fixed sands, 22 \& 24.VI.2004 (S. Belokobylskij) (all in ZISP).
Description. Female. Body length 1.9-2.0 $\mathrm{mm}$; fore wing length $1.5 \mathrm{~mm}$.

Head distinctly transverse, its width 2.2 times median length (dorsal view), 1.2-1.25 times width of mesoscutum. Head behind eyes (dorsal view) distinctly and roundly narrowed. Transverse diameter of eye (dorsal view) about twice as long as temple (2.0-2.7 times for measure on straight line). Ocelli medium-sized, in triangle with base 1.2-1.3 times its sides. POL 1.6-2.0 times OD, almost equal to OOL. Furrow between hind ocelli absent. Eye glabrous, 1.4 times as high as broad. Malar space height $0.25-0.3$ times height of eye, 0.7-0.9 times basal width of mandible. Hypostomal tooth (lateral view) long and pointed. Face width almost equal to height of eye and 2.2-2.3 times height of face; face without middle ridge. Distance between antennal sockets 2.5-3.0 times diameter of socket, about 3.0 times distance between socket and eye. Malar suture absent. Clypeus weakly convex, about 3.0 times as wide as high, its ventral margin weakly concave. Mandible broad basally, uniformly narrowed towards apex, dorsal tooth distinctly longer than ventral tooth. Maxillary palpi about half as long as head height (without mandible).

Antennae rather slender, filiform, 19-segmented, 0.75-0.8 times as long as body. Scapus $1.5-1.6$ times as long as its maximum width. First flagellar segment 2.2-2.5 times as long as its apical width, 1.0-1.1 times as long as second segment. Median segments 2.0-2.2 times as long as wide. Penultimate segment 2.1-2.2 times as long as wide. Last segment pointed apically and with short spine.

Mesosoma. Length 1.45-1.5 times its height, 1.5-1.6 times its width. Notauli distinct on anterior declivity and anterior 0.6 of horizontal part of mesoscutum, almost absent posteriorly, finely crenulate to smooth; medioposterior pit absent. Prescutellar depression rather deep, short, with numerous dense crenulae, $0.15-0.17$ times as long as scutellum. Scutellum convex, rather long and wide, $0.8-0.85$ times as long as wide. Subalar depression deep, narrow, smooth at most part. Sternauli absent. Propodeum without lateral tubercles, propodeal spiracles very small.

Wings. Fore wing 2.4-2.5 times as long as maximum width. Pterostigma subtriangular, wide, 2.4-2.6 times as long as wide. Radial vein 
Fig. 2. Gnaptogaster

- a. Head, front view.

- b. Head, dorsal view.

-c. Basal and apical segments of antenna.

- d. Head, lateral view.

- e. Metasoma, dorsal view. - f. Hind femur. - g. Hind tibia. - h. Fore wing. $-\mathrm{i}$. Hind wing.

Scales: a-d, f, g,

$-X 126 ;$ e -X105;

h, i-X98. levipleuris Tobias.
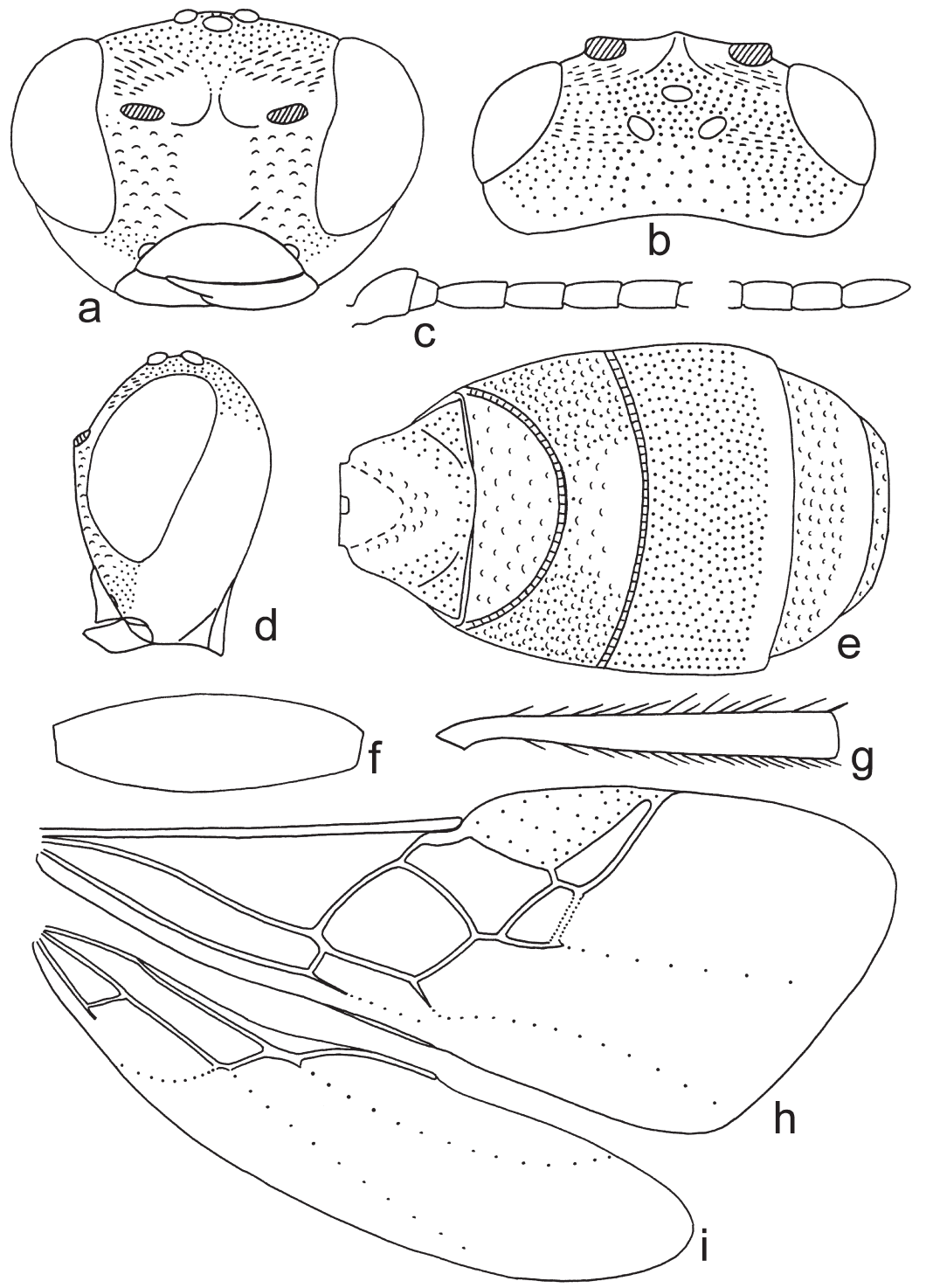

arising before middle of pterostigma. Radial cell very strongly shortened, narrow, wide posteriorly and very narrow anteriorly; metacarpus very short, 0.04-0.06 times as long as pterostigma. Radial vein with 3 abscissae. First radial abscissa $0.15-0.2$ times as long as maximum width of pterostigma. Second radial abscissa 1.2-1.5 times as long as first abscissa, 0.17-0.2 times as long as third abscissa. Third radial abscissa distinctly curved, 1.8 times as long as first radiomedial vein. Second radiomedial cell short, trapeziform, about 0.9 times as long as maximum width. Recurrent vein distinctly antefurcal, 2.5-
3.0 times as long as second abscissa of medial vein. Distance from nervulus to basal vein $0.6-$ 0.75 times nervulus length. Brachial cell widely open posteroapically. Hind wing 3.8-4.2 times as long as wide. First costal abscissa 0.6-0.7 times as long as second abscissa. First abscissa of mediocubital vein 0.7 times as long as second abscissa. Recurrent vein spectral, weakly curved, oblique, directed towards base of wing, shortly antefurcal.

Legs. Hind femur wide, 2.7-3.0 times as long as wide. Hind tibia distinctly widened towards apex, with 3 rather long spines on outer apical 
margin. Hind tarsus narrowed towards apex, about 0.9 times as long as hind tibia. Basitarsus 0.6-0.65 times as long as second-fifth segments combined. Second tarsal segment 0.4-0.45 times as long as basitarsus, 1.3-1.5 times as long as fourth segment, $0.85-0.9$ times as long as fifth segment (without pretarsus).

Metasoma 1.15-1.2 times as long as mesosoma. First tergite without spiracular tubercles, with distinct curved oblique apicolateral furrows, strongly and almost evenly widened from base to apex. Maximum width of first tergite about 3.0 times its minimum width; length 0.6 times its apical width. Second suture deep, narrow and finely crenulate. Basal area of second tergite distinctly delineated by deep and narrow furrow, medially almost as long as half of tergite. Median length of second tergite $0.65-0.7$ times its basal width, 1.0 1.2 times length of third tergite. Ovipositor sheath short, 0.55 times as long as first tergite, $0.15-0.16$ times as long as mesosoma, 0.08 times as long as fore wing.

Sculpture and pubescence. Vertex densely granulate at most part, finely granulate posteriorly; frons finely granulate-coriaceous with fine oblique striation partly, smooth medially; face finely coriaceous, almost smooth widely medially; temple mostly smooth. Sides of pronotum almost smooth, rugose-granulate anteriorly and partly below, without groove posteriorly. Mesoscutum and scutellum smooth, mesoscutum finely punctulate anteriorly. Mesopleura entirely smooth. Metapleura mostly smooth, rugulose posteriorly. Propodeum smooth anteromedially, densely punctulate and partly coriaceous in posterolateral 0.3 . First metasomal tergite very finely coriaceous to smooth or only smooth in wide median part, densely and distinctly granulate-coriaceous in postero-lateral corners. Second tergite densely granulate-coriaceous, almost smooth or finely coriaceous medially; basal area sometimes very finely coriaceous to smooth. Third tergite entirely densely and more or less distinctly granulate. Fourth and fifth tergites densely and finely granulate-coriaceous; posterior margins of third-fifth tergites smooth. Vertex with rather dense short semi-erect setae. Frons bare, but with several setae laterally. Face with sparse erect short setae, widely bare medially. Mesoscutum mostly bare, with dense short setae anteriorly, laterally and medioposteriorly. Hind tibia dorsally with short, rather dense and semierect setae.

Colour. Body black, metasoma dark reddish brown, second tergite laterally yellowish brown or reddish brown. Antennae black, 2 basal segments brownish yellow, but infuscate apically. Palpi yellow. Tegulae brownish yellow. Legs brownish yellow, hind coxa darker, all fifth tarsal segments almost black. Fore wing hyaline. Pterostigma brownish yellow or yellow, infuscate anteriorly.

Male (first record). Body length $2.1 \mathrm{~mm}$; fore wing length $1.6 \mathrm{~mm}$. Transverse diameter of eye 2.6 times as long as temple (3.0 times for measure on straight line). Antennae weakly setiform, 23segmented, as long as body. First flagellar segment twice as long as its apical width. Mesoscutum with shallow wide and short pit medioposteriorly, glabrous but partly sparsely setose around pit. Pterostigma of fore wing 2.1 times as long as wide. Radial vein arising almost from middle of pterostigma. Distance from nervulus to basal vein 0.8 times nervulus length. Metasoma almost as long as mesosoma. Frons entirely densely granulate. Propodeum very finely punctulate posterolaterally. First tergite densely granulate-coriaceous, only coriaceous in wide medioanterior half. Metasoma black. Two basal segments of antenna dark brown dorsally, yellowish brown ventrally. Legs yellowish brown, hind coxa, apical half of hind tibia and hind tarsus at most part infuscate. Otherwise similar to female.

Diagnosis. The differences of $G$. levipleuris Tobias from G. mongolica Tobias and the new species described above are indicated in the key.

Remarks. This species was described based on 2 females (not males as indicated in original description: Tobias 1986: 94). The male of this species, firstly collected in Astrakhan Province, has a peculiar feature (unknown in females), namely a shallow mesoscutal pit. Valuable diagnostic characters of this species, not recorded before, are the pointed hypostomal flange (lateral view) and curved shallow subapical lateral furrows on the first tergite.

Distribution. Kazakhstan, Russia (first record). 


\section{Key to species of the genus Gnaptogaster Tobias}

1. Hypostomal tooth of head (lateral view) distinct and pointed (Fig. 2d). First metasomal tergite with distinct and curved apicolateral oblique furrows (Fig. 2e). Last radial abscissa more or less distinctly convexly curved (Fig. $2 \mathrm{~h})$. Sides of pronotum without posterior vertical furrow. Body length 1.9-2.1 mm. - Kazakhstan (north-east), Russia (south-east of the European part) G. levipleuris Tobias

- Hypostomal tooth of head (lateral view) absent (Fig. 1c). First metasomal tergite without apicolateral oblique furrows (Fig. 1i). Last radial abscissa almost straight or sometimes more or less weakly S-curved (Fig. 1j). Sides of pronotum with posterior vertical furrow (Fig. 1e)

2. Radial vein with 2 abscissae (Fig. 1j). Last radial abscissa almost straight and slender. Second radiomedial cell subtriangular (Fig. 1j). Pterostigma yellow. Basal area of second tergite about as long as half of tergite (Fig. 1i). Metasoma entirely finely but distinctly sculptured (Fig. 1i). Mesoscutum medioposteriorly with several short and sparse setae. Mesosoma of female mostly light reddish brown. Body length 1.8-2.1 mm. - Russia (southeast of the European part)

G. astrachanica sp.n.

- Radial vein with 3 abscissae. Last radial abscissa S-curved and thick. Second radiomedial cell trapeziform. Pterostigma brown. Basal area of second tergite shorter than half of tergite. Metasoma almost entirely smooth. Mesoscutum medioposteriorly with numerous, rather long and dense setae. Mesosoma of female black. Body length $2.1 \mathrm{~mm}$. - Mongolia

G. mongolica Tobias

Acknowledgements. The present work was partly supported by the Russian Foundation for Basic Research (grant No. 04-04-48018).

\section{References}

Achterberg, C. van. 1983: Revisionary notes on the subfamily Gnaptodontinae, with description of eleven new species (Hymenoptera, Braconidae). - Tijdschr. Entomol. 126(1/2): 25-57.

Achterberg, C. van 1988: A new species of the genus Gnamptodon from Italy (Hymenoptera: Braconidae). — Entomol. Bericht. Amsterdam 48(10): 159-161.

Belokobylskij, S. A. 1987: Subfamily Gnaptodontinae (Hymenoptera, Braconidae) of the USSR Far East. In.: Taxonomy of the insects of Siberia and Far East of the USSR: 78-84. [In Russian].

Belokobylskij, S. A. 1993: New taxonomic data on the braconid fauna (Hymenoptera, Braconidae) of Vietnam. - Russ. Entomol. J. 2(2): 37-67.

Belokobylskij, S. A. 1998: Subfam. Gnamptodontinae In: Lehr P. A. (ed.), Key to insects of the Russian Far East. Neuropteroidea, Mecoptera, Hymenoptera [Opredelitel' nasekomykh Dal'nego Vostoka Rossii. Setchatokryloobraznye, skorpionnitzy, pereponchatokrylye]. Vladivostok: Dal'nauka 4(3): 159-162. [In Russian].

Belokobylskij, S.A. 1999: New genera of the subfamilies Rhyssalinae, Exothecinae and Gnamptodontinae from the Old World (Hymenoptera: Braconidae). Zoosyst. Ross. 8(1): 155-169.

Belokobylskij, S. A. \& Tobias, V. I. 1998: Introduction. In: Lehr P. A. (ed.), Key to insects of the Russian Far East. Neuropteroidea, Mecoptera, Hymenoptera [Opredelitel' nasekomykh Dal'nego Vostoka Rossii. Setchatokryloobraznye, skorpionnitzy, pereponchatokrylye]. Vladivostok: Dal'nauka 4(3): 8-26. [In Russian].

Chen, X., Whitfield, J. B. \& He, J. 2002: The discovery of the genus Gnamptodon Haliday (Hymenoptera: Braconidae) in China, with description of one new species. - Pan-Pacific Entomol. 78(3): 184-187.

Fischer, M. 1965: Die Opiinae der nearktischen Region (Hymenoptera, Braconidae). II Teil. — Polsk. Pismo Entomol. 35(1): 3-212.

Fischer, M. 1967: Die amerikanischen Arten der Gattungen Euopius, Gnaptodon und Pseudognaptodon (Hymenoptera; Braconidae). — Beit. Entomol. 17(5/8): 959-976.

Tobias, V. I. 1976: Gnaptogaster mongolica gen. et sp. n. from a new tribe of braconids (Hym., Braconidae, Opiinae) and some problems of the phylogeny and evolution of this family. - In: Kerzhner I. M. (ed.), Insects of Mongolia [Nasekomye Mongolii] 4: 315-321. [In Russian].

Tobias, V. I. 1986: Subfam Gnaptodontinae. - In: Medvedev G.S. (ed.), Key to insects of the European part of the USSR. Hymenoptera [Opredelitel' nasekomykh evropeyskoy chasti SSSR. Pereponchatokrylye]. Leningrad: Nauka 3(4): 85-94. [In Russian].

Tobias, V. I. \& Saidov, N. Sh. 1997: Two new species of braconid wasps (Hymenoptera, Braconidae) from Tajikistan. — Entomol. Obozr. 76(1): 210-212 [In Russian]. 